\title{
Arginine Catabolic Mobile Element Is Associated With Low Antibiotic Resistance and Low Pathogenicity in Staphylococcus epidermidis From Neonates
}

\author{
HILDEGUNN N. GRANSLO, CLAUS KLINGENBERG, ELIZABETH G. A. FREDHEIM, ARILD RØNNESTAD, TOM E. MOLLNES,
} AND TROND FLÆGSTAD

\begin{abstract}
Department of Pediatrics [H.N.G., C.K., E.G.A.F., T.F.], University of Tromsф, N-9037 Troms $\phi$, Norway; Department of Pediatrics [C.K., T.F.], University Hospital of North Norway, N-9038 Tromsø, Norway; Department of Pediatrics [A.R.], Oslo University Hospital, Rikshospitalet, N-0027 Oslo, Norway; Department of Immunology and Transfusion Medicine [T.E.M.], Nordland Hospital, N-8092 Bodø, Norway; Department of Medical Biology [T.E.M.], University of Troms $\phi$, Troms $\phi$, Norway
\end{abstract}

\begin{abstract}
The arginine catabolic mobile element (ACME) in Staphylococci encodes several putative virulence factors. ACME appears to have been transferred from Staphylococcus epidermidis into Staphylococcus aureus and is strongly associated with the epidemic and virulent $S$. aureus USA300. We sought to determine the distribution of ACME in $128 \mathrm{~S}$. epidermidis blood culture isolates from neonates and to assess ACME's impact on antibiotic resistance, biofilm production, invasive capacity, and host inflammatory response. ACME was detected in 15/64 (23\%) invasive blood culture isolates and 26/64 (40\%) blood culture contaminants $(p=0.02)$. ACME-positive $S$. epidermidis isolates displayed less antibiotic resistance $(p<0.001)$ and were collected from more mature neonates $(p=0.001)$. Biofilm production was more prevalent among ACMEnegative isolates (61/87) compared with ACME positive $(18 / 41 ; p=$ $0.004)$. Among the 64 children considered having an invasive infection, ACME did not influence the maximum C-reactive protein level. In an in vitro whole-blood sepsis model, there were no differences in the inflammatory response between ACME-positive and ACMEnegative isolates. We conclude that ACME in S. epidermidis from neonates was associated with less antibiotic resistance and also does not seem to be associated with increased pathogenicity. (Pediatr Res 68: 237-241, 2010)
\end{abstract}

Ctaphylococcus epidermidis is a frequent cause of late onset $\checkmark$ sepsis in preterm neonates $(1,2)$. The major virulence factor of $S$. epidermidis is biofilm formation on polymer surfaces, i.e. indwelling central venous lines (3). A wide range of other putative virulence factors, e.g. surface proteins (4), phenol soluble modulins (PSM), and poly- $\gamma$-glutamic acid $(3,5-7)$, have also been identified. However, their clinical relevance is yet to be proven.

The arginine catabolic mobile element (ACME) is a genomic island in Staphylococci that may contribute to enhanced pathogenicity. It was first discovered in the community associated methicillin-resistant Staphylococcus aureus (CAMRSA) USA300 strain and the biofilm-negative S. epidermidis strain ATCC12228 (8). ACME contains two characteristic

Received January 27, 2010; accepted May 17, 2010.

Correspondence: Claus Klingenberg, M.D., Ph.D., Department of Pediatrics, University Hospital of North Norway, N-9038 Troms $\varnothing$, Norway; e-mail: claus.klingenberg@unn.no

Supported by Grants from Northern Norway Regional Health Authority, Fredriksens legat, and the University of Tromsø. gene clusters (the arc-operon and the opp3-operon) that are homologs of virulence determinants in other bacterial species. The arc-operon, a characteristic cluster of six genes, encodes several enzymes in the arginine deiminase catabolic pathway, a putative virulence pathway that convert L-arginine to carbon dioxide, carbamoyl ornithine, ammonia, and ATP $(9,10)$. The opp3-operon encodes an oligopeptide permease system. Similar opp-operons in other bacterial species have a wide array of functions including pheromone transport, chemotaxis, and expression of virulence determinants (8). ACME has been divided into three allotypes (8-11). ACME-I, first found in $S$. aureus USA300, possess the arc-operon and the opp3-operon. ACME-II, first found in S. epidermidis ATCC12228, consists mainly of the arc-operon. ACME-III has only the opp3operon and lacks the arc-operon. All three allotypes have been detected in S. epidermidis (11).

ACME is associated with the geographically widespread $S$. aureus clone USA300 and is believed to be a survival factor for this species $(8,12)$. One hypothesis is that a decrease in the host inflammatory response is mediated by arginine deiminase inhibition of mononuclear cells (13) and through a depletion of L-arginine, the substrate for nitric oxide production (8). Previous studies have shown a high prevalence of ACME among $S$. epidermidis isolates, indicating that ACME might confer an advantage also to this species $(8,9,11)$.

To our knowledge, no studies have evaluated the inflammatory response to $S$. epidermidis with regard to the presence or absence of ACME. First, the aim of this study was to evaluate the presence of all three ACME allotypes in $S$. epidermidis blood culture isolates obtained from neonates and second, to assess antibiotic resistance and compare different components of the inflammatory response in ACME-positive and ACME-negative isolates.

\footnotetext{
Abbreviations: ACME, arginine catabolic mobile element; CoNS, coagulase-negative Staphylococci; CRP, C-reactive protein; MLST, multilocus sequence typing; MRSA, methicillin-resistant Staphylococcus aureus; PFGE, pulsed field gel electrophoresis; SCC, staphylococcal chromosome cassette
} 


\section{MATERIALS AND METHODS}

A collection of 128 S. epidermidis blood culture isolates obtained from patients admitted to the neonatal intensive care unit at Oslo University Hospital, Rikshospitalet, were investigated. Blood culture isolates that were accompanied by clinical signs of sepsis in a neonate older than $72 \mathrm{~h}$ of age, and with a C-reactive protein (CRP) level $>10 \mathrm{mg} / \mathrm{L}$ were considered as true invasive isolates. All other isolates were considered contaminants. Clinical and microbiologic data, including molecular genetic typing [pulsed field gel electrophoresis (PFGE) and multilocus sequence typing (MLST)] have been published previously $(14,15)$. PFGE patterns were analyzed by GelCompar II version 2.5 (Applied Maths, Belgium). Isolates with $\geq 95 \%$ similarity were considered to be indistinguishable strains and isolates with $\geq 80 \%$ similarity were considered to be related strains $(14,15)$.

We determined the minimal inhibitory concentration (MIC) values of oxacillin, gentamicin, fusidic acid, clindamycin, and vancomycin with Etest (AB Biodisk, Solna, Sweden) and ciprofloxacin by disk diffusion test. Antibiotic susceptibility was interpreted according to the EUCAST clinical breakpoints (16). Semiquantitative determination of biofilm production in microtitre plates was performed and interpreted as previously described $(14,17) . S$. epidermidis ATCC 35984 (RP62A) and S. epidermidis ATCC 12228 served as positive and negative controls, respectively. We performed PCRs using previously reported primers for (i) the methicillin resistance gene (mecA) (18), (ii) one central aminoglycoside resistance gene $\left[\operatorname{aac}\left(6^{\prime}\right)-I e-\operatorname{aph}\left(2^{\prime \prime}\right)-I a\right](19)$, (iii) a marker of the ica-operon encoding biofilm accumulation (icaD) (20), and (iv) ACME- $\operatorname{arcA}$ and ACME-opp3 (8). Purified PCR products of two ACME-opp3 and two ACME- $\operatorname{arcA}$ positive strains, respectively, were sequenced (BigDye Terminator, version 3.1; Applied Biosystems, Warrington) and analyzed on an ABI Prism 377 sequence analyzer (Applied Biosystems, Foster City, CA). To assess a possible association between ACME and staphylococcal chromosome cassette (SCC) elements in S. epidermidis, all ACME-positive isolates were further analyzed with a multiplex PCR (eight loci A-H), as previously described (21).

We selected eight of the ACME-positive and eight of the ACME-negative isolates for further experiments using a whole-blood model to assess in vitro inflammatory response. All 16 isolates were genetically distinguishable by PFGE. We minimized differences between the groups in potentially confounding factors, notably variables, which were previously shown to influence inflammatory response (biofilm production and methicillin resistance) (14). We also had equal numbers of invasive and noninvasive strains in each group. Each experiment was run in two parallels with blood from one healthy volunteer. Blood was collected into sterile polypropylene tubes $(4.5 \mathrm{~mL}$ Nunc cryotubes; Nalgene NUNC, Roskilde, Denmark) containing $50 \mu \mathrm{g} / \mathrm{mL}$ lepirudin (Refludan, Pharmion ApS, Copenhagen, Denmark). Lepirudin, a recombinant hirudin analog with highly specific thrombin inhibitory activity, was used as anticoagulant because it has minimal influence on complement activation (22). The blood samples were obtained immediately before each experiment and kept at $37^{\circ} \mathrm{C}$. Bacteria were added to create a blood-bacteria solution containing $\sim 10^{8}$ colony forming units $(\mathrm{CFU}) / \mathrm{mL}$, verified by spreading diluted inoculum on blood-agar plates and determining CFU count. Oxidative burst and CD11b expression, indicating leukocyte activation, was analyzed in a flow cytometer. Six hundred microliters of the blood-bacteria solution was used for both these analyses after an incubation period of $10 \mathrm{~min}$ at $37^{\circ} \mathrm{C}$. Median fluorescence intensity (MFI) of oxidative-bursted leukocytes was measured according to the manufacturer's instructions with the Phagoburst kit (ORPEGEN, Heidelberg, Germany) on a FACSAria flow cytometer (Becton Dickinson, San Jose, CA). CD11b expression was measured on a FACSCalibur flow cytometer (Becton Dickinson, San Jose, CA) as described previously (22).
A multiplex assay (Bioplex cytokine assay; Bio-Rad, Hercules, CA) was used to quantify proinflammatory cytokines and chemokines (TNF- $\alpha$, IL- $1 \beta$, MIP- $1 \alpha$, IL-6, and IL-8) according to the manufacturer's instructions. In short, $1.8 \mathrm{~mL}$ of the blood-bacteria solution was transferred to segments of polyvinyl chloride (PVC) tubing (length $30 \mathrm{~cm}$, internal diameter $3 \mathrm{~mm}$, Mediplast, Malmø, Sweden). Each segment was closed end-to-end and formed small loops. The loops were rotated slowly in an incubator at $37^{\circ} \mathrm{C}$ for $3 \mathrm{~h}(22,23)$. After $3 \mathrm{~h}$, plasma was separated, and proinflammatory cytokines and chemokines were analyzed. Soluble terminal C5b-9 complement complex (TCC) was analyzed in plasma by an ELISA kit (HK328; Hycult Biotechnologies, Uden, Netherlands) using the same technique as for the cytokine assay but with a 30-min incubation. EDTA was added (final concentration of $10 \mathrm{mM}$ ) immediately after incubation.

The committee for human medical research ethics, Region North in Norway, approved the collection of bacterial isolates, analysis of patient data, and collection of blood for the immune response study. Informed written consent was obtained from each blood donor.

The Mann-Whitney test was used to compare the differences between continuous variables without normal distribution and the $\chi^{2}$ test or Fisher's exact, as appropriate, were used for dichotomous variables. Among the neonates with S. epidermidis sepsis (invasive isolates), we performed a linear multivariable regression analysis to identify bacterial and clinical variables potentially influencing CRP, as a marker of inflammatory response. CRP values were $\log$ transformed to reduce skewness. Two-sample $t$ test was used for the in vitro inflammatory response study. All analyses were two tailed, and $p<0.05$ was considered to be significant. Statistical analysis was performed with SPSS for Windows software version 16.0 (Chicago, IL).

\section{RESULTS}

Sixty-four isolates were considered as true invasive isolates, and 64 isolates were considered contaminants based on our predefined criteria (Table 1). Forty-one of 128 (32\%) isolates were ACME positive; 20 ACME-I, 17 ACME-II, and 4 ACME-III. Table 1 shows the distribution of ACME-positive and ACME-negative isolates among invasive and contaminant $S$. epidermidis blood culture isolates. Twenty-six of 64 (40\%) blood culture contaminants were ACME positive, whereas only 15 of $64(23 \%)$ invasive isolates were ACME positive $(p=0.02)$. ACME-positive blood culture contaminants were collected from a group of neonates that had a higher birth weight and GA compared with neonates with ACME-negative contaminants (Table 1). There was no difference in the inflammatory response (CRP) in patients with ACME-positive invasive isolates compared with patients with ACME-negative invasive isolates (Table 1). A post hoc linear multivariable regression analysis adjusting for possible confounders (biofilm production, methicillin resistance, GA, and age at onset infection) also failed to show any influence of ACME on neonatal inflammatory response (data not shown).

Table 1. S. epidermidis blood culture isolates; ACME and relation to clinical data

\begin{tabular}{|c|c|c|c|c|c|c|}
\hline & \multicolumn{3}{|c|}{ Invasive $(n=64)$} & \multicolumn{3}{|c|}{ Contaminants $(n=64)$} \\
\hline & $\begin{array}{l}\text { ACME-positive } \\
\quad(n=15)\end{array}$ & $\begin{array}{l}\text { ACME-negative } \\
\quad(n=49)\end{array}$ & $p$ & $\begin{array}{l}\text { ACME-positive } \\
\quad(n=26)\end{array}$ & $\begin{array}{l}\text { ACME-negative } \\
\quad(n=38)\end{array}$ & $p$ \\
\hline \multicolumn{7}{|l|}{ Clinical data } \\
\hline GA (wk) & $\begin{array}{c}28 \\
(27-40)\end{array}$ & $\begin{array}{c}27 \\
(26-32)\end{array}$ & 0.36 & $\begin{array}{c}40 \\
(31-40)\end{array}$ & $\begin{array}{c}28 \\
(26-35)\end{array}$ & 0.001 \\
\hline Birth weight $(\mathrm{g})$ & $\begin{array}{c}1120 \\
(750-3410)\end{array}$ & $\begin{array}{c}960 \\
(701-1783)\end{array}$ & 0.36 & $\begin{array}{c}2810 \\
(1351-3670)\end{array}$ & $\begin{array}{c}970 \\
(790-2230)\end{array}$ & 0.001 \\
\hline $\mathrm{CRP}(\mathrm{mg} / \mathrm{L})$ & $\begin{array}{c}54 \\
(18-114)\end{array}$ & $\begin{array}{c}47 \\
(34-82)\end{array}$ & 0.92 & $\mathrm{NP}^{*}$ & NP & \\
\hline
\end{tabular}

Data are presented as median (interquartile range).

* NP, Not presented (data not presented as CRP $<10 \mathrm{mg} / \mathrm{L}$ is one of the criteria defining an isolate as a contaminant). 


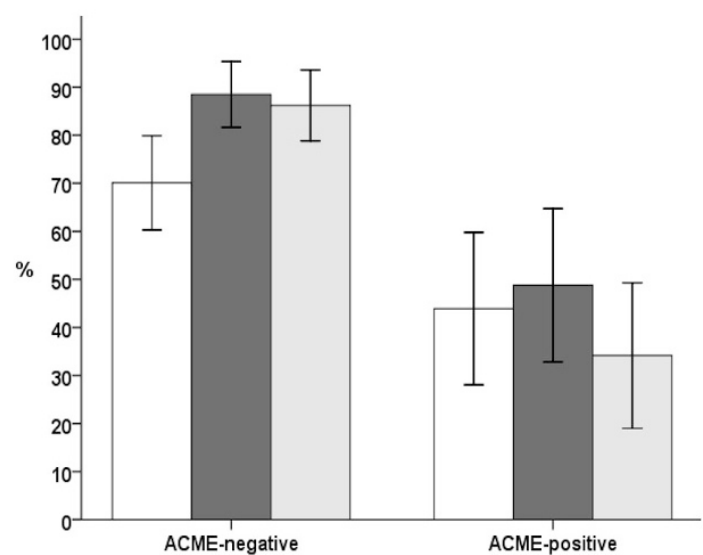

Figure 1. Biofilm production and methicillin- and gentamicin resistance among ACME-positive and ACME-negative blood culture isolates $(n=128)$ obtained from neonates. $\square$, biofilm positive; $\square$, methicillin resistance; and $\square$, gentamicin resistance. $T$ bars represent $95 \%$ confidence intervals.

Molecular typing with PFGE showed two large clusters of related S. epidermidis isolates (15 strains PFGE-type 1 and 35 strains PFGE-type 27) comprising 50 of the 128 neonatal blood culture isolates. Molecular typing with MLST confirmed that these cluster isolates were related and that the vast majority of them belonged to a clonal complex, designated ST2 (15). Only two of 50 cluster isolates were ACMEpositive. The other 39 ACME-positive isolates were evenly distributed among 78 sporadic, nonrelated S. epidermidis isolates. Sequencing ACME-arcA and ACME-opp3 from four isolates verified that the gene products were the same as those previously published $(8,11)$. SCCmec typing of the 41 ACMEpositive isolates revealed 2 isolates with SCCmec type IV, 33 isolates with nontypeable SCC elements, and in 6 isolates no SCC elements were detected.

Among all 128 isolates, methicillin resistance $(p<0.001)$, gentamicin resistance $(p<0.001)$, and biofilm production $(p=0.004)$ were more prevalent among ACME-negative isolates compared with ACME-positive isolates (Fig. 1). None of the strains were resistant to vancomycin. To exclude impact of clustering, we did a subgroup analysis of the 78 sporadic nonrelated isolates. ACME-negative isolates still displayed significant higher methicillin resistance (29/39 versus 19/ $39, p=0.035)$ and gentamicin resistance (27/39 versus $13 / 39, p=0.003$ ) than ACME-positive isolates, but there were no longer significant difference in biofilm production (data not shown). Among sporadic isolates, 20/39 ACMEnegative and 14/39 ACME-positive isolates were considered invasive ( $p=0.17)$.

Results from the whole-blood model of inflammatory response to $S$. epidermidis are shown in Table 2. We found no significant differences between ACME-positive and -negative strains for a broad range of inflammatory response pathways.

\section{DISCUSSION}

We found a high prevalence of ACME in S. epidermidis isolates from neonates. Our finding is in line with the study by Diep et al. (8), in which $67 \%$ (18/27) of S. epidermidis isolates from nostrils were ACME positive, and Miragaia et al. (11), in
Table 2. Inflammatory response measurements for eight ACME-positive and eight ACME-negative S. epidermidis isolates in a human whole-blood model

\begin{tabular}{cccc}
\hline & $\begin{array}{c}\text { ACME } \\
\text { positive }\end{array}$ & $\begin{array}{c}\text { ACME } \\
\text { negative }\end{array}$ & $p$ \\
\hline Oxidative burst & & & \\
Granulocyte burst* & $51(14)$ & $43(18)$ & 0.323 \\
Granulocyte (MFI) & $307(111)$ & $238(140)$ & 0.296 \\
Monocyte burst* & $28(14)$ & $21(12)$ & 0.303 \\
Monocyte (MFI) & $139(64)$ & $104(38)$ & 0.207 \\
CD11b expression & & & \\
Granulocytes (MFI) & $49,183(12093)$ & $53,553(9016)$ & 0.426 \\
Monocyte (MFI) & $12,976(4931)$ & $12,920(5504)$ & 0.983 \\
TCC (Au/mL) & $113(23)$ & $111(21)$ & 0.852 \\
Cytokines & & & \\
TNF- $\alpha(\mathrm{pg} / \mathrm{mL})$ & $41,713(11862)$ & $47,627(13690)$ & 0.371 \\
MIP-1 $\alpha(\mathrm{pg} / \mathrm{mL})$ & $908(228)$ & $1,104(259)$ & 0.131 \\
IL-8 $(\mathrm{pg} / \mathrm{mL})$ & $7,758(2846)$ & $9,174(4765)$ & 0.483 \\
IL-6 $(\mathrm{pg} / \mathrm{mL})$ & $2,994(1296)$ & $3,365(1291)$ & 0.575 \\
IL-1 $\beta(\mathrm{pg} / \mathrm{mL})$ & $3,294(1709)$ & $4,259(2 / 996)$ & 0.189 \\
\hline
\end{tabular}

All 16 isolates are biofilm producers (icaD-positive) and oxacillin resistant (mecA-positive).

MFI, median fluorescence intensity; TCC, terminal complement complex. Data presented as mean (SD).

* Percent cells burst/total cells.

which $51 \%(65 / 127)$ of S. epidermidis isolates from different sources and widespread geographical origin were ACMEpositive. A recent study also showed a high prevalence (48\%) of ACME in Staphylococcus haemolyticus isolates (24). In this study, a higher prevalence of ACME was found in $S$. epidermidis isolates considered as contaminants (most likely derived from the skin) compared with invasive disease causing isolates. It has been suggested that L-arginine catabolism contributes to optimize staphylococcal life on the acidic human skin, which may explain the high prevalence of ACME in typically skin colonizing coagulase-negative Staphylococci (CoNS), such as S. epidermidis and S. haemolyticus $(10,24)$. Transfer of ACME from CoNS to the USA300 MRSA clone may also explain why USA300 frequently is colonizing skin regions (e.g. axilla and inguinal) not previously considered as typical niches for MRSA colonization $(11,25)$.

In $S$. aureus, the role of ACME on virulence is unclear. In a rabbit MRSA bacteremia model, the ACME deletion mutant showed attenuated bacterial infectivity (9), whereas in a rat model of MRSA pneumonia, ACME had no impact on virulence (26). One epidemiologic study of S. epidermidis found no association between ACME and disease causing isolates, but clinical details were not presented (11). Our study did not identify any association between presence of ACME and invasive neonatal $S$. epidermidis infections. We noted that the mean GA of infants with contaminant isolates was higher than of infants with invasive blood culture isolates ( $p=0.02)$, most likely reflecting that preterm infants have an impaired immune system and higher risk acquiring invasive $S$. epidermidis infections (27). ACME was frequently found in contaminant isolates from mature term-born babies, and we speculate that ACME in S. epidermidis may be considered as an indicator of benign, skin flora isolates.

To our knowledge, no previous data has been presented on the correlation between ACME and inflammatory response to 
S. epidermidis. We have previously shown that biofilmpositive CoNS sepsis isolates induced a lower neonatal CRP response than biofilm-negative isolates. In contrast, methicillin resistance was associated with higher inflammatory response, most likely because of the delay in adequate antibiotic therapy (14). In our analysis of 64 episodes of S. epidermidis neonatal sepsis (invasive isolates), the presence or absence of ACME did not influence neonatal inflammatory response, even after adjusting for possible confounders. However, these results should be interpreted with caution as the limited number of ACME-positive isolates and high standard deviations of CRP levels only gave us a power to detect $>50 \%$ difference in CRP levels. In the human whole-blood model, we also found no differences in a broad range of inflammatory responses between ACME-positive and ACME-negative isolates. This whole-blood model is developed specifically for studying the interaction of pathways of inflammation $(22,28)$ and is considered a robust model for detecting differences in inflammatory response. Thus, in our study, ACME in $S$. epidermidis did not have any obvious impact on the host inflammatory response.

In S. aureus, ACME-positive isolates cluster in major MRSA-clonal lineages (9). Molecular typing of S. epidermidis blood culture isolates in our study revealed a different epidemiologic pattern, where ACME was associated with a high clonal diversity. In contrast, ACME was rarely present in the local endemic $S$. epidermidis clone affecting more immature infants in the neonatal intensive care unit (15). A similar high clonal diversity was also found among 88 ACME-positive $S$. haemolyticus isolates in a study from China (24). Miragaia et $a l$., in contrast, found that in geographically diverse S. epidermidis isolates, the vast majority of ACME containing isolates belonged to a large clonal complex. There is now compelling evidence that ACME is widely distributed among different CoNS-species, and that CoNS may act as a reservoir for ACME being transferred to specific clonal lineages of $S$. aureus $(8,11,24)$.

Studies in $S$. aureus show that ACME is often integrated in the bacterial chromosome adjacent to a SCCmec IV element. It has been suggested that the physical linkage between ACME and SCCmec type IV leads to enhanced fitness by coselecting for beta-lactam resistance and possibly other multidrug resistant determinants $(9,12,29)$. In contrast to the findings in S. aureus, and in line with Miragaia et al. (11), we found no association between ACME and SCCmec type IV in $S$. epidermidis. Furthermore, we actually observed a significant lower rate of methicillin and gentamicin resistance among ACME-positive versus ACME-negative $S$. epidermidis isolates. The recombinases used for integration of ACME and SCCmec into the bacterial chromosome are able to integrate these two genetic elements independently $(9,11)$, and different selection mechanisms may apply for $S$. aureus and $S$. epidermidis. Avoidance of carrying large genetic elements encoding antibiotic resistance or biofilm production may theoretically increase biologic fitness and thereby improve the capacity of colonizing $S$. epidermidis strains to grow and survive within a host.
There are limitations to our study. The isolates included are from a single Norwegian neonatal unit. Thus, it may be difficult to generalize our findings to S. epidermidis strains in other countries. Distinguishing between "true" S. epidermidis sepsis and blood culture contamination is also notoriously difficult, especially in neonates. However, our definition of neonatal $S$. epidermidis sepsis is in line with others (1), and it includes clinical signs of sepsis and a significant increase in host inflammatory response judged by CRP. We studied selected parts of the host inflammatory response in vitro and some parameters of interest could have been missed. However, we argue that our fairly broad approach cover central parts of host inflammatory response. Other investigators have used isogenic wild-type/ACME-deletion mutant pair for comparison of virulence $(9,26)$. In the human whole-blood model, we used eight ACME-positive and eight ACME-negative $S$. epidermidis blood culture isolates matched for different putative virulence factors, but it is possible that other confounding virulence factors could have influenced the inflammatory response. The concentration of $S$. epidermidis used in our whole-blood model is high. However, this concentration is similar to lethal and sublethal doses of Staphylococci used in primate models of sepsis $(30,31)$. During sepsis, bacteria often demonstrate massive growth within the first 12-24 $\mathrm{h}$ after entering the blood stream (32). The natural bactericidal activity of blood then subsequently reduces the viability of organisms that can be recovered from blood cultures (33). Quantitative neonatal blood cultures often reveal CoNS concentration around $10^{3} \mathrm{CFU} / \mathrm{mL}$. However, sepsis studies assessing total bacterial DNA load have shown that the difference between dead and live bacteria may be three to four orders of magnitude, and that both dead and live bacteria induce the innate immune system $(32,34)$. We assessed the inflammatory response during a short 3 -h period and, thus, argue that the bacterial concentration used in our experiments was adequate.

\section{CONCLUSIONS}

We conclude that ACME in S. epidermidis from neonates was associated with a lower prevalence of antibiotic resistance and biofilm production compared with the ACME-negative isolates. ACME was more commonly found in S. epidermidis blood culture isolates considered as contaminants and isolates obtained from mature babies. We found neither in vivo nor in vitro association between ACME in S. epidermidis and increased host inflammatory response. We suggest that the presence of ACME in S. epidermidis does not seem to lead to those isolates being more harmful.

Acknowledgments. We thank the following for excellent technical assistance: Research Laboratory at Nordland Hospital in Bod $\varnothing$, Flow Cytometry Core Facility at the University of Troms $\varnothing$, Lillian Marstein at the MRSA Reference Laboratory, St. Olavs Hospital in Trondheim, and Vivian Berg, Goran Kauric, and Yngve Figenschau, all at the University of Troms $\varnothing$. We thank our two participants who provided blood for the inflammatory response model. We also thank Johanna 
E. Sollid and Kevin I. Wheeler for critically commenting on results and manuscript.

\section{REFERENCES}

1. Stoll BJ, Hansen N, Fanaroff AA, Wright LL, Carlo WA, Ehrenkranz RA, Lemons JA, Donovan EF, Stark AR, Tyson JE, Oh W, Bauer CR, Korones SB, Shankaran S, Laptook AR, Stevenson DK, Papile LA, Poole WK 2002 Late-onset sepsis in very low birth weight neonates: the experience of the NICHD Neonatal Research Network. Pediatrics 110:285-291

2. Ronnestad A, Abrahamsen TG, Medbo S, Reigstad H, Lossius K, Kaaresen PI, Egeland T, Engelund IE, Irgens LM, Markestad T 2005 Late-onset septicemia in a Norwegian national cohort of extremely premature infants receiving very early full human milk feeding. Pediatrics 115:e269-e276

3. Otto M 2008 Staphylococcal biofilms. In: Romeo T (ed) Bacterial Biofilms. Springer, Berlin, Germany, pp 207-228

4. Bowden MG, Chen W, Singvall J, Xu Y, Peacock SJ, Valtulina V, Speziale P, Hook M 2005 Identification and preliminary characterization of cell-wall-anchored proteins of Staphylococcus epidermidis. Microbiology 151:1453-1464

5. Otto M 2004 Virulence factors of the coagulase-negative Staphylococci. Front Biosci 9:841-863

6. Kocianova S, Vuong C, Yao YF, Voyich JM, Fischer ER, Deleo FR, Otto M 2005 Key role of poly-gamma-DL-glutamic acid in immune evasion and virulence of Staphylococcus epidermidis. J Clin Invest 115:688-694

7. Liles WC, Thomsen AR, O'Mahony DS, Klebanoff SJ 2001 Stimulation of human neutrophils and monocytes by staphylococcal phenol-soluble modulin. J Leukoc Biol 70:96-102

8. Diep BA, Gill SR, Chang RF, Phan TH, Chen JH, Davidson MG, Lin F, Lin J, Carleton HA, Mongodin EF, Sensabaugh GF, Perdreau-Remington F 2006 Complete genome sequence of USA300, an epidemic clone of community-acquired methicillin-resistant Staphylococcus aureus. Lancet 367:731-739

9. Diep BA, Stone GG, Basuino L, Graber CJ, Miller A, Etages SA, Jones A, Palazzolo-Ballance AM, Perdreau-Remington F, Sensabaugh GF, Deleo FR, Chambers HF 2008 The arginine catabolic mobile element and staphylococcal chromosomal cassette mec linkage: convergence of virulence and resistance in the USA300 clone of methicillin-resistant Staphylococcus aureus. J Infect Dis 197:1523-1530

10. Diep BA, Otto M 2008 The role of virulence determinants in community-associated MRSA pathogenesis. Trends Microbiol 16:361-369

11. Miragaia M, de Lencastre H, Perdreau-Remington F, Chambers HF, Higashi J, Sullam PM, Lin J, Wong KI, King KA, Otto M, Sensabaugh GF, Diep BA 2009 Genetic diversity of arginine catabolic mobile element in Staphylococcus epidermidis. PLoS One 4:e7722

12. Goering RV, McDougal LK, Fosheim GE, Bonnstetter KK, Wolter DJ, Tenover FC 2007 Epidemiologic distribution of the arginine catabolic mobile element among selected methicillin-resistant and methicillin-susceptible Staphylococcus aureus isolates. J Clin Microbiol 45:1981-1984

13. Degnan BA, Palmer JM, Robson T, Jones CE, Fischer M, Glanville M, Mellor GD, Diamond AG, Kehoe MA, Goodacre JA 1998 Inhibition of human peripheral blood mononuclear cell proliferation by Streptococcus pyogenes cell extract is associated with arginine deiminase activity. Infect Immun 66:3050-3058

14. Klingenberg C, Aarag E, Ronnestad A, Sollid JE, Abrahamsen TG, Kjeldsen G, Flaegstad T 2005 Coagulase-negative staphylococcal sepsis in neonates: association between antibiotic resistance, biofilm formation and the host inflammatory response. Pediatr Infect Dis J 24:817-822

15. Klingenberg C, Ronnestad A, Anderson AS, Abrahamsen TG, Zorman J, Villaruz A, Flaegstad T, Otto M, Sollid JE 2007 Persistent strains of coagulase-negative Staphylococci in a neonatal intensive care unit: virulence factors and invasiveness. Clin Microbiol Infect 13:1100-1111

16. European Committee on Antimicrobial Susceptibility Testing (EUCAST) 2010 Clinical breakpoints. Available at: http://www.eucast.org/clinical_breakpoints. Accessed April 20, 2010
17. Christensen GD, Simpson WA, Younger JJ, Baddour LM, Barrett FF, Melton DM, Beachey EH 1985 Adherence of coagulase-negative Staphylococci to plastic tissue culture plates: a quantitative model for the adherence of Staphylococci to medical devices. J Clin Microbiol 22:996-1006

18. Predari SC, Ligozzi M, Fontana R 1991 Genotypic identification of methicillinresistant coagulase-negative Staphylococci by polymerase chain reaction. Antimicrob Agents Chemother 35:2568-2573

19. Klingenberg C, Sundsfjord A, Ronnestad A, Mikalsen J, Gaustad P, Flaegstad T 2004 Phenotypic and genotypic aminoglycoside resistance in blood culture isolates of coagulase-negative Staphylococci from a single neonatal intensive care unit, 1989-2000. J Antimicrob Chemother 54:889-896

20. de Silva GD, Kantzanou M, Justice A, Massey RC, Wilkinson AR, Day NP, Peacock SJ 2002 The ica operon and biofilm production in coagulase-negative Staphylococci associated with carriage and disease in a neonatal intensive care unit. J Clin Microbiol 40:382-388

21. Oliveira DC, de Lencastre H 2002 Multiplex PCR strategy for rapid identification of structural types and variants of the mec element in methicillin-resistant Staphylococcus aureus. Antimicrob Agents Chemother 46:2155-2161

22. Mollnes TE, Brekke OL, Fung M, Fure H, Christiansen D, Bergseth G, Videm V, Lappegard KT, Kohl J, Lambris JD 2002 Essential role of the C5a receptor in $E$ coli-induced oxidative burst and phagocytosis revealed by a novel lepirudin-based human whole-blood model of inflammation. Blood 100:1869-1877

23. Sprong T, Moller AS, Bjerre A, Wedege E, Kierulf P, van der Meer JW, Brandtzaeg P, van Deuren M, Mollnes TE 2004 Complement activation and complementdependent inflammation by Neisseria meningitidis are independent of lipopolysaccharide. Infect Immun 72:3344-3349

24. Pi B, Yu M, Chen Y, Yu Y, Li L 2009 Distribution of the ACME-arcA gene among methicillin-resistant Staphylococcus haemolyticus and identification of a novel ccr allotype in ACME-arcA-positive isolates. J Med Microbiol 58:731-736

25. Miller LG, Diep BA 2008 Clinical practice: colonization, fomites, and virulence: rethinking the pathogenesis of community-associated methicillin-resistant Staphylococcus aureus infection. Clin Infect Dis 46:752-760

26. Montgomery CP, Boyle-Vavra S, Daum RS 2009 The Arginine Catabolic Mobile Element (ACME) is not associated with enhanced virulence in experimental invasive disease caused by the community-associated methicillin-resistant Staphylococcus aureus (CA-MRSA) genetic background USA300. Infect Immun 77:2650-2656

27. Strunk T, Richmond P, Simmer K, Currie A, Levy O, Burgner D 2007 Neonatal immune responses to coagulase-negative Staphylococci. Curr Opin Infect Dis 20:370-375

28. Lappegard KT, Christiansen D, Pharo A, Thorgersen EB, Hellerud BC, Lindstad J, Nielsen EW, Bergseth G, Fadnes D, Abrahamsen TG, Hoiby EA, Schejbel L, Garred P, Lambris JD, Harboe M, Mollnes TE 2009 Human genetic deficiencies reveal the roles of complement in the inflammatory network: lessons from nature. Proc Natl Acad Sci U S A 106:15861-15866

29. Ellington MJ, Yearwood L, Ganner M, East C, Kearns AM 2008 Distribution of the ACME-arcA gene among methicillin-resistant Staphylococcus aureus from England and Wales. J Antimicrob Chemother 61:73-77

30. Zimecki M, Artym J, Kocieba M, Weber-Dabrowska B, Borysowski J, Gorski A 2010 Prophylactic effect of bacteriophages on mice subjected to chemotherapyinduced immunosuppression and bone marrow transplant upon infection with Staphylococcus aureus. Med Microbiol Immunol 199:71-79

31. Sauer M, Altrichter J, Kreutzer HJ, Logters T, Scholz M, Noldge-Schomburg G, Schmidt R, Mitzner SR 2009 Extracorporeal cell therapy with granulocytes in a pig model of Gram-positive sepsis. Crit Care Med 37:606-613

32. Ovstebo R, Brandtzaeg P, Brusletto B, Haug KB, Lande K, Hoiby EA, Kierulf P 2004 Use of robotized DNA isolation and real-time PCR to quantify and identify close correlation between levels of Neisseria meningitidis DNA and lipopolysaccharides in plasma and cerebrospinal fluid from patients with systemic meningococcal disease. J Clin Microbiol 42:2980-2987

33. Buttery JP 2002 Blood cultures in newborns and children: optimising an everyday test. Arch Dis Child Fetal Neonatal Ed 87:F25-F28

34. Hackett SJ, Guiver M, Marsh J, Sills JA, Thomson AP, Kaczmarski EB, Hart CA 2002 Meningococcal bacterial DNA load at presentation correlates with disease severity. Arch Dis Child 86:44-46 